\title{
Application of intraoperative B-mode ultrasound and shear wave elastography for glioma grading
}

\author{
Lu Yin ${ }^{1}$, Linggang Cheng ${ }^{1}$, Fumin Wang ${ }^{1,2}$, Xueli Zhu ${ }^{1}$, Yue Hua ${ }^{1}$, Wen $\mathrm{He}^{1}$ \\ ${ }^{1}$ Department of Ultrasound, Beijing Tiantan Hospital, Capital Medical University, Beijing, China; ${ }^{2}$ Department of Ultrasound, Peking University \\ First Hospital, Beijing, China
}

Correspondence to: Wen He. Department of Ultrasound, Beijing Tiantan Hospital, Capital Medical University, No.119 West Section of South 4th Ring Road, Fengtai District, Beijing, 100070, China. Email: hewen@bjtth.org.

\begin{abstract}
Background: To evaluate the value of intraoperative B-mode ultrasound and shear wave elastography (SWE) in differentiating low-grade and high-grade gliomas.

Methods: A total of 172 patients with glioma were examined by B-mode ultrasound to obtain a tumor sonogram. Intraoperative SWE was performed on 52 patients to obtain Young's modulus values of peritumor tissue and tumor tissue, and the differences in conventional B-mode signs and Young's modulus values of gliomas of different grades were then compared. The diagnostic performance of SWE in glioma grading was assessed by receiver operating characteristic (ROC) curve analysis, and the intra- and interobserver reliability of SWE was analyzed by the intraclass correlation coefficient (ICC).

Results: For B-mode ultrasound, patient age, cystic degeneration, and peritumor edema were independent risk factors for high-grade glioma $(\mathrm{P}<0.05, \mathrm{OR}>1)$. For $\mathrm{SWE}$, Young's modulus values of peritumor tissue, low-grade glioma, and high-grade glioma tissues were $8.20(7.50,9.70) \mathrm{kPa}, 19.65(15.30,24.75) \mathrm{kPa}$, and $9.55(8.50,13.80) \mathrm{kPa}$, respectively. The area under the ROC curve for the diagnosis of high-grade glioma by SWE was 0.859 (95\% CI: $0.758-0.961, \mathrm{P}<0.05$ ), and the optimal cutoff value was $12.1 \mathrm{kPa}$, with $89.3 \%$ sensitivity and $75.0 \%$ specificity. The intra- and interobserver reliability of SWE in grading gliomas was excellent, with ICCs ranging from 0.921 to 0.965 .

Conclusions: High-grade glioma is associated with significantly more severe necrotic cystic degeneration and peritumoral edema on B-mode ultrasound and lower stiffness on SWE. Further, SWE exhibits excellent intra- and interobserver reliability. Intraoperative B-mode ultrasound combined with SWE helps differentiate different grades of gliomas.
\end{abstract}

Keywords: Shear wave elastography (SWE); ultrasound; glioma; intraoperative imaging; surgery

Submitted Dec 17, 2020. Accepted for publication Mar 12, 2021.

doi: $10.21037 /$ qims-20-1368

View this article at: http://dx.doi.org/10.21037/qims-20-1368

\section{Introduction}

Glioma is the most common neuroepithelial tumor, accounting for about $40 \%$ of primary intracranial tumors (1), and those of a high-grade are characterized by rapid progression, severe symptoms, and poor quality of life. The 5-year survival rate of glioblastoma (GBM) is less than $5 \%$, with a median survival of approximately 14 months (2,3), and while surgical resection is the current primary treatment approach, the postoperative residual tumor is an important factor in the prognosis of patients $(4,5)$. Low-grade glioma refers to grade I-II, and high-grade glioma refers to grade III-IV based on the World Health Organization (WHO) criteria (6). The selection of surgical procedures is partly determined by the grade of glioma and by assessing the complexity of surgical resection (7-10). To ensure patient management is continuously improved, it is extremely important to implement accurate glioma 
classification strategies that rely on intraoperative imaging.

Preoperative magnetic resonance imaging (MRI) is the primary diagnostic modality for the classification of glioma and residual tumors (11). However, it shows the poor diagnostic performance when tumor calcification and hemorrhagic necrosis are present, and its sensitivity for the diagnosis of high-grade glioma is highly variable, ranging from 55.1-83.9\% (12). On this basis, MRI cannot be used as an objective method for diagnostic grading before surgery.

Intraoperative B-mode ultrasound is a safe, convenient, and effective imaging tool for intraoperative monitoring that has been widely used in neurosurgery. Studies have shown that intraoperative ultrasound monitoring can help neurosurgeons locate intraoperative tumors, guide surgery, and identify residual tumors (13-15), and its application has also been found to help improve the postoperative quality of life of glioma patients $(16,17)$. However, there are few studies on the classification of glioma by intraoperative B-mode ultrasound.

As an emerging ultrasonic technique, shear wave elastography (SWE) can differentiate tissues by assessing their stiffness quantitatively according to Young's modulus and can provide more supplementary information to improve diagnostic accuracy. SWE tracks the propagation of the shear wave generated by the acoustic radiation force and shows that the shear wave travels faster through stiffer tissues. Some studies have reported different SWE patterns for different types of brain tumors $(18,19)$, but at present, the use of SWE to predict the grade of glioma has seldomly been reported.

This study aimed to assess the value of intraoperative B-mode ultrasound and SWE in the diagnostic grading of glioma by analyzing gliomas' characteristics using these methods and providing a theoretical basis for identifying residual tumors using SWE.

\section{Methods}

The Ethics Committee approved this study of the Beijing Tiantan Hospital (KY2018-097-02), and all participating patients or their guardians provided written informed consent.

\section{Research population}

Patients with single supratentorial superficial glioma who were scheduled to undergo brain tumor removal at the Neurosurgery Department of our hospital between
December 2018 and June 2019 were enrolled in this study. Patients with multiple gliomas, tumor recurrence, chemotherapy, or other types of intracranial tumors such as meningioma or neurofibroma were excluded. All enrolled patients underwent MRI examination before surgery and were diagnosed with intracranial glioma by postoperative pathological examination.

\section{$B$-mode and SWE examinations}

A Hitachi Noblus scanner (Hitachi, Japan) with a C42 convex array probe (frequency: 3-12 MHz) and an Aixplorer scanner (SuperSonic Imagine, France) with an SMC123 convex array probe (frequency: 3-12 MHz) were used to acquire B-mode and SWE images, respectively.

B-mode images were acquired after craniotomy and before dural opening. The probe was protected with sterile sheets and positioned perpendicularly over the dura while images were acquired. The size, morphology, border, echo, cystic area, degree of edema, and calcification of tumors were recorded. The diameter of the tumor was measured at the largest part of the tumor, and tumor size was divided into $<3 \mathrm{~cm}, 3-5 \mathrm{~cm}$, and $>5 \mathrm{~cm}$ categories (20). The extent of peripheral edema was also assessed at the largest part of the tumor, with mild edema defined as $\leq 2 \mathrm{~cm}$ and severe edema as $>2 \mathrm{~cm}$ (21).

The SWE examination was conducted after the B-mode scan. This saw the dura opened, and patients treated with cerebrospinal fluid drainage and mannitol infusion to reduce cranial pressure and avoid excessive bulging of brain tissue, which may influence SWE measurement accuracy. The probe was coated with ultrasound transmission gel, protected with sterile sheets, placed perpendicularly over the dura, then placed lightly on the brain's surface with minimal pressure. The tumor was focused and centered in the B-mode display; the probe kept steady to obtain a clear B-mode image and then switched to SWE mode. The SWE sampling frame was controlled at a size of $2-4 \mathrm{~cm}$ and depth of $1-4 \mathrm{~cm}$ to adjust to the solid center of the tumor, and no additional pressure was applied by the operator during the whole process. When the color stably filled more than $80 \%$ of the sampling frame area, the image was frozen and played back to obtain a qualified SWE image for measurement. A region of interest (ROI) that was filled with color was chosen, then Young's modulus was measured automatically (color bar: 0-55 kPa). When the color filled the ROI and the minimum Young's modulus of the ROI was greater than 0 , the measurement was considered successful, and if 
Table 1 Clinical information of the patients and tumor characteristics

\begin{tabular}{|c|c|}
\hline Characteristics & Total $n=172$ \\
\hline Age (years), range (mean $\pm S D$ ) & $18-69(43.7 \pm 15.0)$ \\
\hline \multicolumn{2}{|l|}{ Sex } \\
\hline Male & 108 \\
\hline Female & 64 \\
\hline Tumors size $(\mathrm{mm})$, range (mean $\pm \mathrm{SD})$ & $12-81(41.8 \pm 14.6)$ \\
\hline \multicolumn{2}{|l|}{ Tumors location } \\
\hline Frontal lobe & 78 \\
\hline Parietal lobe & 48 \\
\hline Temporal lobe & 27 \\
\hline Occipital lobe & 19 \\
\hline \multicolumn{2}{|l|}{ Pathology } \\
\hline \multicolumn{2}{|l|}{ Low-grade } \\
\hline WHO Grade I & - \\
\hline Pilocytic astrocytoma & 6 \\
\hline Angiocentric glioma & 1 \\
\hline \multicolumn{2}{|l|}{ WHO Grade II } \\
\hline Diffuse astrocytoma & 30 \\
\hline Oligodendroglioma & 49 \\
\hline \multicolumn{2}{|l|}{ High-grade } \\
\hline WHO Grade III & - \\
\hline Anaplastic astrocytoma & 14 \\
\hline Anaplastic oligodendroglioma & 16 \\
\hline WHO Grade IV & - \\
\hline Glioblastoma & 56 \\
\hline
\end{tabular}

unsuccessful, re-measurement was performed. A patient with five consecutive unsuccessful measurements was excluded from SWE examination, and the average of three measurements of the mean Young's modulus was used for further analysis. SWE examination was performed on both the solid portion of the tumor and the peritumor tissues. Peritumor tissue was defined as tissue that was within $2 \mathrm{~cm}$ of the edge of the tumor and tissue exhibiting edema was avoided. The SWE measurement was performed at the same horizontal position with the tumor.

Two observers performed the SWE examination to assess the reproducibility and reliability of SWE in glioma grading, and following the above protocol; the first SWE examination was performed by observer A after the dura was opened. The second examination was performed by observer B at the same position and depth of the tumor. Then a second SWE examination was performed by observer A. Observer A had 5 years of experience in ultrasonic elastography, and each observer was blind to the other's result and the clinical information of the patients. The agreement between the same observer and between different observers was assessed.

After the operation, tumor tissue was taken for pathologic analysis to determine the glioma's pathological type and grade.

\section{Statistical analysis}

SPSS 20.0 statistical software was used for data analysis (IBM Corp., Armonk, NY, US). According to a normality assessment using the Shapiro-Wilk test, all measurement data were expressed as the mean \pm standard deviation or the median and interquartile range. $t$-tests or nonparametric rank-sum tests were used to compare two groups of measurement data, the $\chi^{2}$ test was used for counting data, and logistic regression was used for multifactor analysis. The Kruskal-Wallis test was used to compare multiple measurement data, and post hoc analysis was performed using the Mann-Whitney $U$ test with Bonferroni correction. The diagnostic performance was evaluated by receiver operating characteristic (ROC) curve analysis. Finally, an optimal cutoff point was determined to obtain a numerical value that would allow discrimination between low-grade and highgrade gliomas, and the intra- and interobserver reliability was assessed using the intraclass correlation coefficient (ICC). The effect size, 95\% confidence intervals (CIs), and $\mathrm{P}$ values were calculated with a $\mathrm{P}$ value $<0.05$ considered to be statistically significant.

\section{Results}

All patients enrolled in the study underwent tumor resection, and a summary of their clinical characteristics and the tumors is shown in Table 1 . We performed a B-mode examination on 172 patients (64 women and 108 men; mean age: $43.7 \pm 15.0$ years old), and SWE on 52 patients, including 28 patients with low-grade glioma and 24 with high-grade glioma. The Young's modulus value of the peritumor tissue could not be measured in several patients because of limitations related to the bone flap size and 

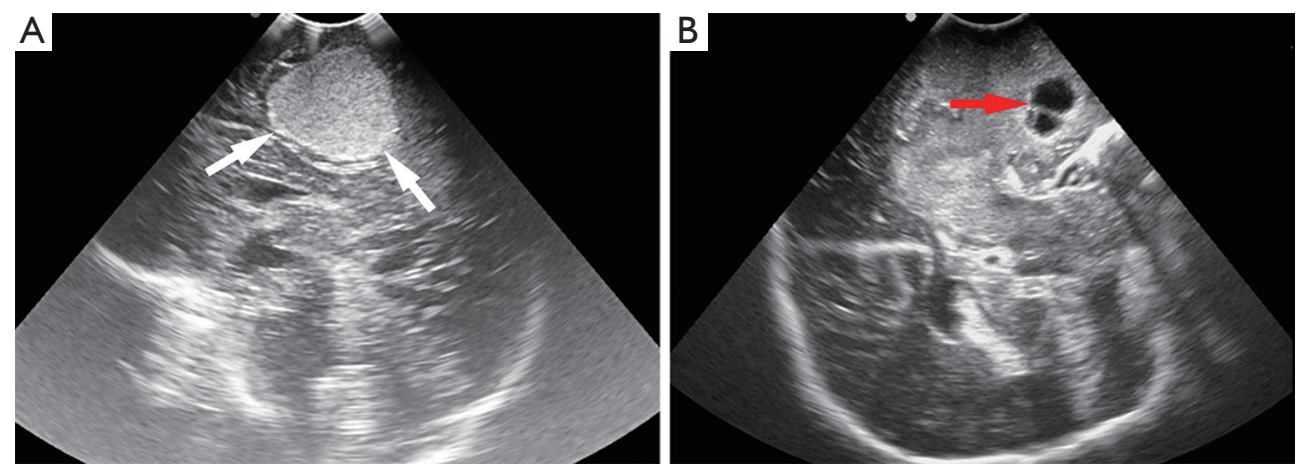

Figure 1 Representative B-Mode images. (A) A hyperechoic tumor with a clear boundary determined to be a low-grade glioma with the white arrows indicating the tumor. (B) A hyperechoic tumor with an unclear boundary and cystic degeneration determined to be high-grade glioma with the red arrow indicating cystic degeneration.

tumor location, which resulted in only 33 patients included for further analysis.

\section{B-mode examination}

Glioma has various B-mode manifestations, which can be hyperechoic, isoechoic, or mixed echogenicity with regular or irregular morphology, and clear or unclear boundaries, while some may have necrotic cystic degeneration. Representative B-mode images are shown in Figure 1.

Data on the age, sex, and B-mode manifestations of patients with low- and high-grade glioma were analyzed, and the results revealed significant differences in age, tumor morphology, clear boundaries, tumor echo, cystic degeneration, degree of peripheral edema, and uniform internal echo (all $\mathrm{P}<0.05$ ) (Table 2). Univariate analysis was used for screening, and eligible factors were included in the multivariate logistic regression analysis, which showed patient age, cystic tumor degeneration, and peripheral edema were independent risk factors for high-grade glioma $(\mathrm{OR}>1, \mathrm{P}<0.05)$ (Figure 2).

\section{SWE examination}

The Young's modulus values of peritumor tissue, lowgrade glioma, and high-grade glioma were $8.20(7.50,9.70)$ $\mathrm{kPa}, 19.65(15.30,24.75) \mathrm{kPa}$, and $9.55(8.50,13.80) \mathrm{kPa}$, respectively, with significant differences found among the groups $(\mathrm{P}<0.05)($ Table 3). The representative SWE images are shown in Figure 3. The post boc analysis revealed that pairwise comparisons of the different Young's modulus values for peritumor tissue, low-grade glioma, and high- grade glioma also showed significant differences (all $\mathrm{P}<0.05$ ) (Table 3). The Young's modulus value increased in the order of peritumor tissue, high-grade glioma, and low-grade glioma, and a comparison of Young's modulus between lowand high-grade gliomas, excluding GBM, is listed in Table 4.

ROC curves were used to analyze the diagnostic performance of SWE for high-grade glioma, and the area under the curve was 0.859 (95\% CI: 0.758 to $0.961, \mathrm{P}<0.05$ ) (Figure 4). The best cutoff value was $12.1 \mathrm{kPa}$, with $89.3 \%$ sensitivity and $75.0 \%$ specificity.

Finally, as the duration of surgery, the intra- and the interobserver reliability of SWE were determined for only 12 patients. The ICCs for intraobserver and interobserver reliability ranged only from 0.921 to 0.965 . The details are shown in Table 5.

\section{Discussion}

This study verified the value of intraoperative B-mode ultrasound and SWE in the diagnostic grading of glioma and indicated that patient age, cystic tumor degeneration, and peripheral edema were independent risk factors for high-grade glioma. High-grade gliomas had a lower Young's modulus than low-grade gliomas, and the best cutoff value for the diagnosis of high-grade glioma in SWE was $12.1 \mathrm{kPa}$.

A total of 172 patients with glioma were observed for routine B-mode ultrasonographic manifestations, including tumor size, echo intensity, boundary, internal echo uniformity, peripheral edema degree, cystic degeneration, and calcification. The results revealed significant differences between low-grade glioma and high-grade glioma in terms 
Table 2 Comparison of B-mode signs between low-grade and high-grade glioma

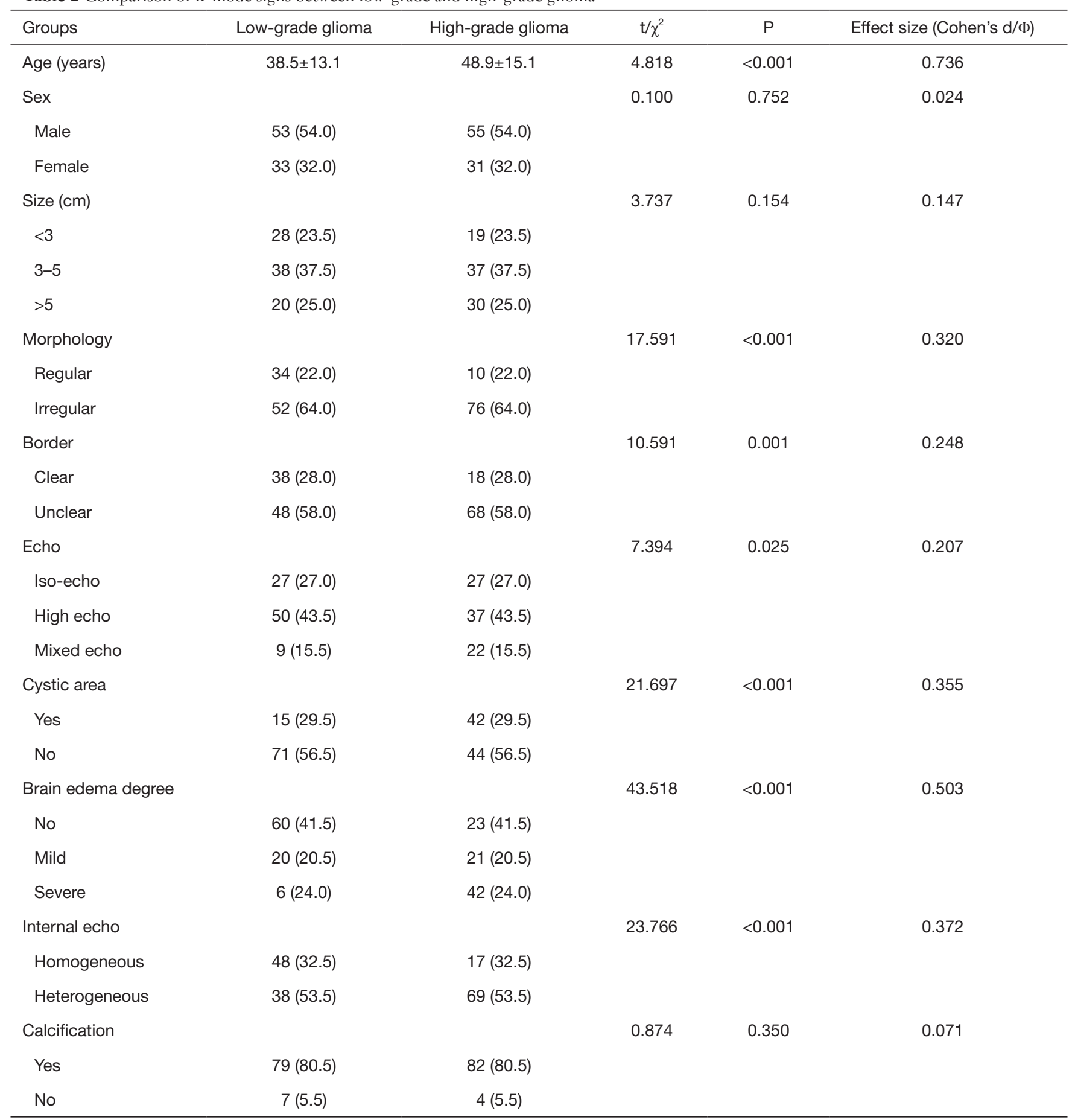

Data are shown as $\mathrm{n}$ (theoretical numbers). 


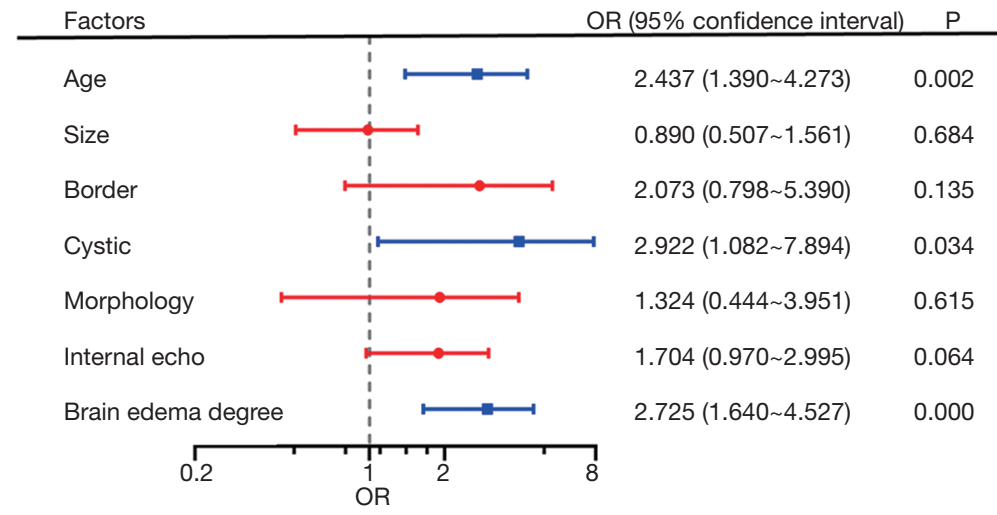

Figure 2 Logistic regression analysis of the independent risk factors for high-grade glioma. Patient age, tumor cystic degeneration, and peripheral edema were found to be independent risk factors for high-grade glioma.

Table 3 Comparison of Young's modulus in groups of peritumor tissue, low-grade, and high-grade glioma

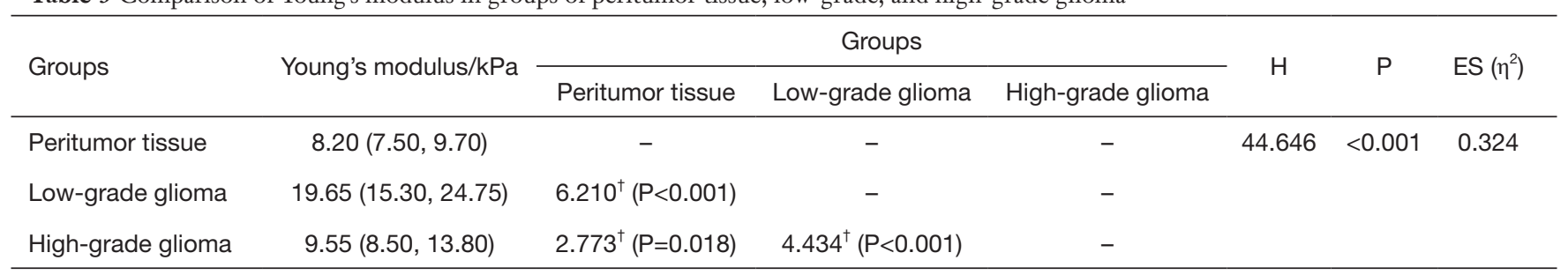

Data are shown as the median and interquartile range. ${ }^{\dagger}$, the testing value by post hoc analysis using the Mann-Whitney $\mathrm{U}$ test. ES, effect size.

of age, tumor morphology, boundaries, tumor echo, cystic degeneration, degree of peripheral edema, and internal echo. Multivariate logistic regression analysis showed that age, cystic tumor degeneration, degree of edema around the tumor, and uneven internal echogenicity of the tumor were independent risk factors for high-grade glioma, which indicated that the older the patient was and the more severe the degree of peritumoral edema, the greater the probability of high-grade glioma. These results are partly consistent with the findings obtained by MRI (22), and they may relate to the growth pattern of high-grade gliomas, which grow rapidly and have an insufficient blood supply, leading to necrosis of the emergent tissues (23). Also, highgrade glioma's growth process often leads to the destruction of the blood-brain barrier and an increase in vascular permeability (24), causing edema in the surrounding brain tissue. Therefore, attention should be paid to these signs during an intraoperative ultrasound examination.

In this study, 52 of 172 patients with glioma were examined by intraoperative SWE, which showed that Young's modulus of low-grade glioma was significantly higher than that of high-grade glioma, which is similar to the results of a previous study (19). This may be related to the pathological characteristics of glioma. Low-grade glioma mostly shows a dense and uniform cell arrangement with less bleeding and necrosis, while the differentiation of high-grade glioma cells is immature, the arrangement is disordered, and there are more bleeding and necrosis areas inside, resulting in the low stiffness of high-grade gliomas. In clinical practice, surgery and postoperative treatment are mainly conducted by neurosurgeons according to the presence of a high-grade or low-grade glioma. However, the ultrasound characteristics of GBM are recognizable for any experienced examiner, and many neurosurgeons are concerned about the differences in SWE characteristics between low-grade and high-grade gliomas, excluding GBM. Our results indicate that Young's modulus of lowgrade gliomas was higher than that of high-grade gliomas, 

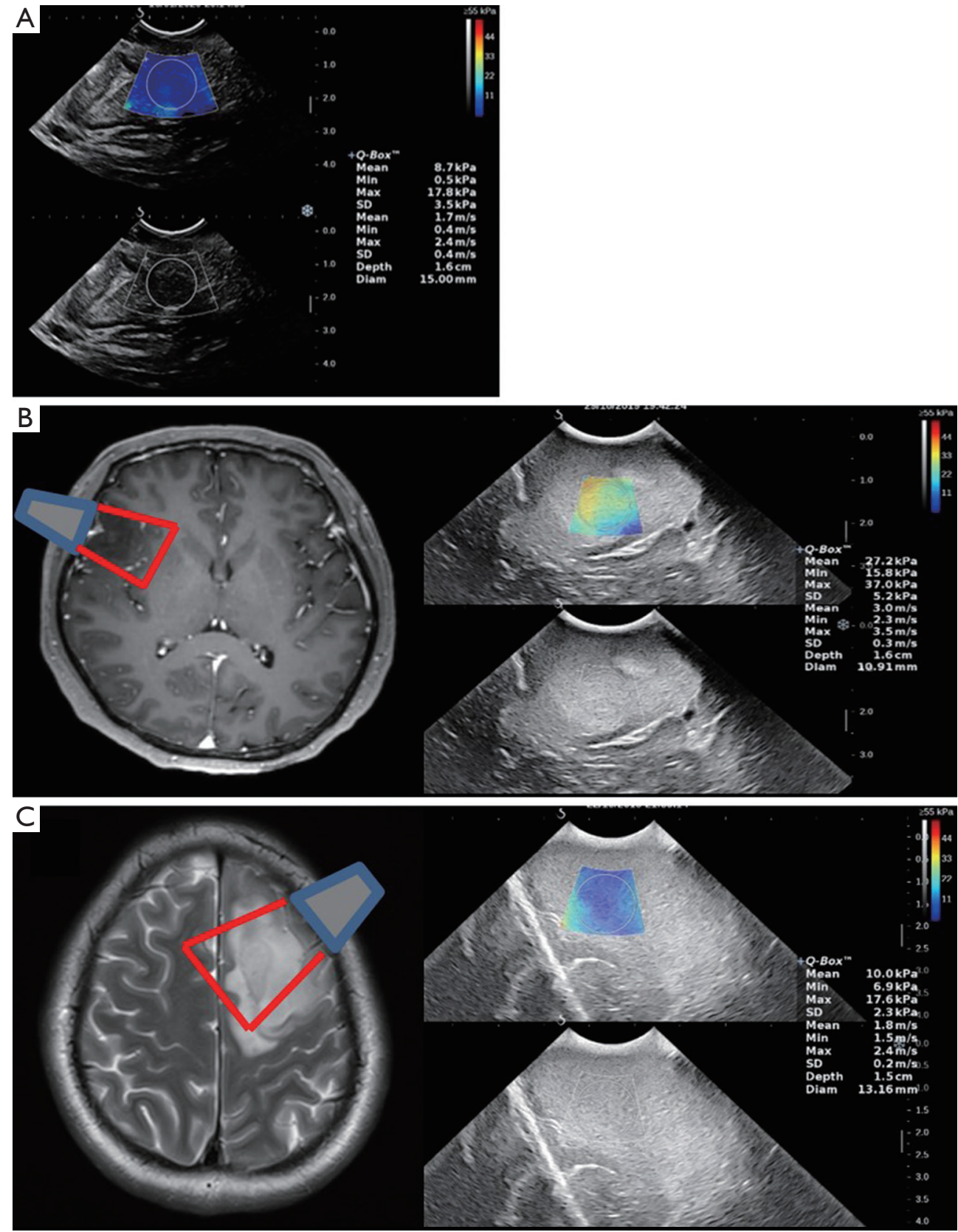

Figure 3 Representative SWE images of peritumor tissue and glioma tissue. (A) peritumor tissue with a Young's modulus of $8.7 \mathrm{kPa}$; (B) low-grade glioma with a Young's modulus of $27.2 \mathrm{kPa}$; (C) high-grade glioma with a Young's modulus of $10.0 \mathrm{kPa}$. The Young's modulus was increased in the order of peritumor tissue, high-grade glioma, and low-grade glioma.

Table 4 Comparison of Young's modulus between low-grade and high-grade glioma, excluding GBM

\begin{tabular}{|c|c|c|c|c|c|}
\hline Grade & Number of cases & Young's modulus/kPa & Z & $\mathrm{P}$ & $\mathrm{ES}\left(\eta^{2}\right)$ \\
\hline High-grade glioma (w/o GBM) & 10 & $12.550(8.425,18.700)$ & & & \\
\hline
\end{tabular}

Data are shown as the median and interquartile range. GBM, glioblastoma; ES, effect size. 


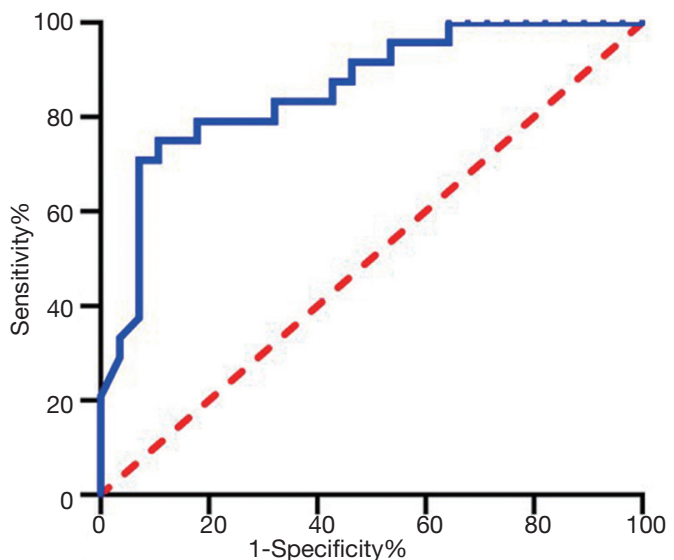

Figure 4 Analysis of the ROC curve of SWE for the diagnosis of high-grade glioma. The area under the curve was 0.859 (95\% CI: 0.758 to 0.961$)$.

Table 5 Agreement assessment of SWE in grading gliomas

\begin{tabular}{l}
$\begin{array}{l}\text { Observers and } \\
\text { groups }\end{array}$ \\
\hline
\end{tabular}

\section{Observer A,}

observation 1
Total $(n=12)$

Low-grade

glioma $(n=7)$

High-grade

glioma $(n=5)$

Observer A, observation 2

Total $(n=12)$

Low-grade

glioma $(n=7)$

High-grade

glioma $(n=5)$

Observer B

Total $(n=12)$

Low-grade

glioma $(n=7)$

High-grade

glioma $(n=5)$
12.500

(10.900-19.775)

18.800

(13.100-25.700)

10.800

(9.400-11.550)
Data are shown as the median and interquartile range. SWE, shear wave elastography; ICC, intraclass correlation coefficient; $\mathrm{Cl}$, confidence interval. excluding GBM. The pathological results were taken as the gold standard in our study, and the area under the ROC curve to analyze the diagnostic performance of SWE in predicting glioma grading was 0.859 . The best cutoff value was $12.1 \mathrm{kPa}$, indicating that tumors with Young's modulus less than $12.1 \mathrm{kPa}$ tended to be high-grade glioma, and those with Young's modulus over $12.1 \mathrm{kPa}$ tended to be lowgrade glioma. Intraoperative pathologic diagnosis, including frozen sections and cytology smears, provides a real-time diagnosis for surgeons, but the heterogeneity of the tumor, accuracy of sampling, and duration of examination limit its diagnostic accuracy, ranging from $71.40 \%$ to $96.06 \%$ (25). As a real-time, quantitative, and objective tool, SWE could provide additional information to obtain a more precise real-time diagnosis for neurosurgeons when distinguishing high-grade glioma from low-grade glioma intraoperatively. SWE also compensates for the deficiency of distinguishing glioma levels by morphological changes and has guiding significance for adjusting the surgical plan and selecting a suitable treatment strategy.

In addition to the grade identification of glioma, the residual tumor size is also of great importance to patient treatment and prognosis. Some reports have shown that patients' postoperative survival is related to the degree of glioma resection (26). Accordingly, identifying residual tumor tissues from normal brain tissue has become the focus and represents a major challenge for an intraoperative ultrasound. In this study, the SWE results revealed that Young's modulus of peritumor tissue was lower than that of glioma tissues, indicating that SWE may be used to identify residual glioma from the surrounding normal tissue and could provide a research direction for intraoperative localization and residual tumor identification. Some studies have even shown that ultrasound elastography helped assess the completeness of resection in epilepsy surgery and brain tumor surgery $(27,28)$. However, a case series study demonstrated that it could not detect boundaries between lesions and normal brain tissue (29). The choice of elastography methods may affect the results because strain elastography requires an external force while SWE does not. Our results show that differences between peritumor tissue and high-grade glioma tissue existed, but these findings were limited. Therefore, distinguishing the two by SWE only may be infeasible and requires further study.

The variation in intra- and interobserver reliability in SWE limits its applicability at present. In this study, the intra- and interobserver reliability was verified by ICCs, 
and the results showed that SWE exhibited satisfactory reliability in determining the stiffness of gliomas of different grades.

This study also has limitations. Firstly, the sample size for the SWE evaluation was small and further studies with larger samples are needed to understand better its value for the intraoperative determination of residual tumor. Secondly, all cases in this study were supratentorial superficial tumors, and Young's modulus of the deep basement nuclear mass was not considered. Finally, contrastenhanced intraoperative ultrasound (CE-IoUS) was not used in our study. The future of intraoperative ultrasound could be revolutionized by the advent of CE-IoUS, which may serve as a surrogate for more expensive intraoperative imaging modalities such as computerized tomography and MRI. Therefore, the future armamentarium for neurooncology surgeons could expand over time as the number of contrast media currently under advanced laboratory testing will continue to increase (30-34). Despite the above deficiencies, intraoperative B-mode ultrasound and SWE are undoubtedly valuable for neurosurgeons due to their ability to distinguish high-grade gliomas from low-grade gliomas.

\section{Conclusions}

High-grade glioma is associated with significantly more severe necrotic cystic degeneration and peritumoral edema on B-mode and lower stiffness on SWE than low-grade glioma. Further, SWE exhibits excellent intraobserver and interobserver reliability. Therefore, intraoperative B-mode ultrasound combined with SWE can provide morphological information of gliomas and enable the assessment of glioma stiffness both objectively and quantitatively, which will help neurosurgeons in grading glioma and adjusting the surgical plan during the operation.

\section{Acknowledgments}

We gratefully thank all participants for their collaboration in this study.

Funding: This work was supported by the National Natural Science Foundation of China (grant number: 81730050), the Youth Project of the Beijing Natural Science Foundation (grant number: 7204255), and the Cultivation Project of the Beijing Municipal Administration of Hospitals (grant number: px2018021).

\section{Footnote}

Conflicts of Interest: All authors have completed the ICMJE uniform disclosure form (available at http://dx.doi. org/10.21037/qims-20-1368). The authors have no conflicts of interest to declare.

Ethical Statement: This prospective study was approved by the Institutional Review Boards of the Beijing Tiantan Hospital (KY2018-097-02) and was conducted in accordance with the Declaration of Helsinki. Written informed consent was obtained from all subjects.

Open Access Statement: This is an Open Access article distributed in accordance with the Creative Commons Attribution-NonCommercial-NoDerivs 4.0 International License (CC BY-NC-ND 4.0), which permits the noncommercial replication and distribution of the article with the strict proviso that no changes or edits are made and the original work is properly cited (including links to both the formal publication through the relevant DOI and the license). See: https://creativecommons.org/licenses/by-nc-nd/4.0/.

\section{References}

1. Luo L, Guan X, Begum G, Ding D, Gayden J, Hasan MN, Fiesler VM, Dodelson J, Kohanbash G, Hu B, Amankulor NM, Jia W, Castro MG, Sun B, Sun D. Blockade of Cell Volume Regulatory Protein NKCC1 Increases TMZInduced Glioma Apoptosis and Reduces Astrogliosis. Mol Cancer Ther 2020;19:1550-61.

2. Ostrom QT, Bauchet L, Davis FG, Deltour I, Fisher JL, Langer CE, Pekmezci M, Schwartzbaum JA, Turner MC, Walsh KM, Wrensch MR, Barnholtz-Sloan JS. The epidemiology of glioma in adults: a "state of the science" review. Neuro Oncol 2014;16:896-913.

3. Shi J, Hou S, Huang J, Wang S, Huan W, Huang C, Liu X, Jiang R, Qian W, Lu J, Wang X, Shi W, Huang R, Chen J. An MSN-PEG-IP drug delivery system and IL13R $\alpha 2$ as targeted therapy for glioma. Nanoscale 2017;9:8970-81.

4. Fukui A, Muragaki Y, Saito T, Maruyama T, Nitta M, Ikuta S, Kawamata T. Volumetric Analysis Using LowField Intraoperative Magnetic Resonance Imaging for 168 Newly Diagnosed Supratentorial Glioblastomas: Effects of Extent of Resection and Residual Tumor Volume on Survival and Recurrence. World Neurosurg 2017;98:73-80.

5. Fujii Y, Muragaki Y, Maruyama T, Nitta M, Saito T, Ikuta 
S, Iseki H, Hongo K, Kawamata T. Threshold of the extent of resection for WHO Grade III gliomas: retrospective volumetric analysis of 122 cases using intraoperative MRI. J Neurosurg 2018;129:1-9.

6. Louis DN, Perry A, Reifenberger G, von Deimling A, Figarella-Branger D, Cavenee WK, Ohgaki H, Wiestler OD, Kleihues P, Ellison DW. The 2016 World Health Organization Classification of Tumors of the Central Nervous System: a summary. Acta Neuropathol 2016;131:803-20.

7. Ganau L, Ligarotti GKI, Ganau M. Predicting complexity of tumor removal and postoperative outcome in patients with high-grade gliomas. Neurosurg Rev 2018;41:371-3.

8. Jeong J, Wang L, Ji B, Lei Y, Ali A, Liu T, Curran WJ, Mao H, Yang X. Machine-learning based classification of glioblastoma using delta-radiomic features derived from dynamic susceptibility contrast enhanced magnetic resonance images: Introduction. Quant Imaging Med Surg 2019;9:1201-13.

9. Goyal P, Kumar Y, Gupta N, Malhotra A, Gupta S, Gupta S, Mangla M, Mangla R. Usefulness of enhancementperfusion mismatch in differentiation of CNS lymphomas from other enhancing malignant tumors of the brain. Quant Imaging Med Surg 2017;7:511-9.

10. Brahimaj BC, Kochanski RB, Pearce JJ, Guryildirim M, Gerard CS, Kocak M, Sani S, Byrne RW. Structural and Functional Imaging in Glioma Management. Neurosurgery 2021;88:211-21.

11. van Dijken BRJ, van Laar PJ, Holtman GA, van der Hoorn A. Diagnostic accuracy of magnetic resonance imaging techniques for treatment response evaluation in patients with high-grade glioma, a systematic review and metaanalysis. Eur Radiol 2017;27:4129-44.

12. Law M, Yang S, Wang H, Babb JS, Johnson G, Cha S, Knopp EA, Zagzag D. Glioma grading: sensitivity, specificity, and predictive values of perfusion MR imaging and proton MR spectroscopic imaging compared with conventional MR imaging. AJNR Am J Neuroradiol 2003;24:1989-98.

13. Ganau L, Paris M, Ligarotti GK, Ganau M. Management of Gliomas: Overview of the Latest Technological Advancements and Related Behavioral Drawbacks. Behav Neurol 2015;2015:862634.

14. Ganau M, Ligarotti GK, Apostolopoulos V. Real-time intraoperative ultrasound in brain surgery: neuronavigation and use of contrast-enhanced image fusion. Quant Imaging Med Surg 2019;9:350-8.

15. Bal J, Camp SJ, Nandi D. The use of ultrasound in intracranial tumor surgery. Acta Neurochir (Wien) 2016;158:1179-85.

16. Garzon-Muvdi T, Kut C, Li X, Chaichana KL. Intraoperative imaging techniques for glioma surgery. Future Oncol 2017;13:1731-45.

17. Mahboob S, McPhillips R, Qiu Z, Jiang Y, Meggs C, Schiavone G, Button T, Desmulliez M, Demore C, Cochran S, Eljamel S. Intraoperative Ultrasound-Guided Resection of Gliomas: A Meta-Analysis and Review of the Literature. World Neurosurg 2016;92:255-63.

18. Cepeda S, Barrena C, Arrese I, Fernandez-Pérez G, Sarabia R. Intraoperative Ultrasonographic Elastography: A Semi-Quantitative Analysis of Brain Tumor Elasticity Patterns and Peritumoral Region. World Neurosurg 2020;135:e258-70.

19. Chauvet D, Imbault M, Capelle L, Demene C, Mossad M, Karachi C, Boch AL, Gennisson JL, Tanter M. In Vivo Measurement of Brain Tumor Elasticity Using Intraoperative Shear Wave Elastography. Ultraschall Med 2016;37:584-90.

20. Li J, Liang R, Song C, Xiang Y, Liu Y. Prognostic and clinicopathological significance of long non-coding RNA in glioma. Neurosurg Rev 2020;43:1-8.

21. Utsuki S, Oka H, Suzuki S, Shimizu S, Tanizaki Y, Kondo K, Tanaka S, Kawano N, Fujii K. Pathological and clinical features of cystic and noncystic glioblastomas. Brain Tumor Pathol 2006;23:29-34.

22. Wu CX, Lin GS, Lin ZX, Zhang JD, Chen L, Liu SY, Tang WL, Qiu XX, Zhou CF. Peritumoral edema on magnetic resonance imaging predicts a poor clinical outcome in malignant glioma. Oncol Lett 2015;10:2769-76.

23. Llaguno-Munive M, León-Zetina S, Vazquez-Lopez I, Ramos-Godinez MDP, Medina LA, Garcia-Lopez P. Mifepristone as a Potential Therapy to Reduce Angiogenesis and P-Glycoprotein Associated With Glioblastoma Resistance to Temozolomide. Front Oncol 2020;10:581814.

24. Lin Zhi-Xiong. Glioma-related edema: new insight into molecular mechanisms and their clinical implications. Chin J Cancer 2013;32:49-52.

25. Mat Zin AA, Zulkarnain S. Diagnostic Accuracy of Cytology Smear and Frozen Section in Glioma. Asian Pac J Cancer Prev 2019;20:321-5.

26. Hervey-Jumper SL, Berger MS. Role of surgical resection in low- and high-grade gliomas. Curr Treat Options Neurol 2014;16:284.

27. Mathon B, Amelot A, Carpentier A, Clemenceau S. Intraoperative real-time guidance using ShearWave 
Elastography for epilepsy surgery. Seizure 2019;71:24-7.

28. Prada F, Del Bene M, Rampini A, Mattei L, Casali C, Vetrano IG, Gennari AG, Sdao S, Saini M, Sconfienza LM, DiMeco F. Intraoperative Strain Elastosonography in Brain Tumor Surgery. Oper Neurosurg (Hagerstown) 2019;17:227-36.

29. Prada F, Gennari AG, Quaia E, D'Incerti L, de Curtis M, DiMeco F, Tringali G. Advanced intraoperative ultrasound (ioUS) techniques in focal cortical dysplasia (FCD) surgery: A preliminary experience on a case series. Clin Neurol Neurosurg 2020;198:106188.

30. Ganau M. Tackling gliomas with nanoformulated antineoplastic drugs: suitability of hyaluronic acid nanoparticles. Clin Transl Oncol 2014;16:220-3.

31. Ganau M, Syrmos NC, D'Arco F, Ganau L, Chibbaro S,

Cite this article as: Yin L, Cheng L, Wang F, Zhu X, Hua Y, $\mathrm{He}$ W. Application of intraoperative B-mode ultrasound and shear wave elastography for glioma grading. Quant Imaging Med Surg 2021;11(6):2733-2743. doi: 10.21037/qims-20-1368
Prisco L, Ligarotti GKI, Ambu R, Soddu A. Enhancing contrast agents and radiotracers performance through hyaluronic acid-coating in neuroradiology and nuclear medicine. Hell J Nucl Med 2017;20:166-8.

32. Gupta N, Grover H, Bansal I, Hooda K, Sapire JM, Anand R, Kumar Y. Neonatal cranial sonography: ultrasound findings in neonatal meningitis-a pictorial review. Quant Imaging Med Surg 2017;7:123-31.

33. Brem S, Henderson F. Commentary: 5-Aminolevulinic Acid and Contrast-Enhanced Ultrasound: The Combination of the 2 Techniques to Optimize the Extent of Resection in Glioblastoma Surgery. Neurosurgery 2020;86:E541-3.

34. Klibanov AL. Ultrasound Contrast: Gas Microbubbles in the Vasculature. Invest Radiol 2021;56:50-61. 